\title{
A novel prognostic index for sporadic Burkitt lymphoma in adult patients: a real-word multicenter study
}

Mei-ting Chen ${ }^{1 \dagger}$, Fei Pan ${ }^{1 \dagger}$, Yung-chang Chen ${ }^{2 \dagger}$, Wei Zhang ${ }^{2 \dagger}$, Hui-juan Lv ${ }^{3 \dagger}$, Zhao Wang ${ }^{1}$, Huang-ming Hong ${ }^{2}$, Xiao-jie Fang ${ }^{1}$, Ya-wen Wang ${ }^{4}$, Tao Pan ${ }^{5}$, Li-qun Zou ${ }^{6}$, Hong-qiang Guo ${ }^{7}, \mathrm{Ke} \mathrm{Xie}^{8}$, Li-min Chen ${ }^{1}$, Xiao-qian Li ${ }^{1}$, Yu-yi Yao ${ }^{1}$, Ze-geng Chen ${ }^{1}$, Hua-wei Weng ${ }^{1}$, Xu-dong Li ${ }^{2}$, Yuan-yuan Shen ${ }^{2}$, Hui Zhou ${ }^{5}$, Hong-wei Xue ${ }^{4}$, Hui-lai Zhang ${ }^{3}$, He Huang ${ }^{1 *}$ and Tong-yu Lin ${ }^{1,2^{*}}$

\begin{abstract}
Background: Adult sporadic Burkitt lymphoma (BL) is a rare but highly aggressive subtype of lymphoma which lacks its own unique prognostic model. Systemic inflammatory biomarkers have been confirmed as prognostic markers in several types of malignancy. Our objective was to explore the predictive value of pretreatment inflammatory biomarkers and establish a novel, clinically applicable prognostic index for adult patients with sporadic BL.

Methods: We surveyed retrospectively 336 adult patients with newly diagnosed sporadic BL at 8 Chinese medical centers and divided into training cohort $(n=229)$ and validation cohort $(n=107)$. The pretreatment inflammatory biomarkers were calculated for optimal cut-off value. The association between serum biomarkers and overall survival (OS) was analyzed by Kaplan-Meier curves and Cox proportional models. The risk stratification was defined based on normal LDH level, Ann Arbor stage of I and completely resected abdominal lesion or single extra-abdominal mass $<10 \mathrm{~cm}$.
\end{abstract}

Results and conclusions: Univariate and multivariate analyses revealed that platelets $<254 \times 10^{9} / \mathrm{L}$, albumin $<40 \mathrm{~g} / \mathrm{L}$, lactate dehydrogenase $\geq 334 \mathrm{U} / \mathrm{L}$ independently predicted unfavorable OS. We used these data as the basis for the prognostic index, in which patients were stratified into Group 1 (no or one risk factor), Group 2 (two risk factors), or Group 3 (three risk factors), which were associated with 5 -year OS rates of 88.1, 72.4, and 45\%, respectively. In the subgroup analysis for high-risk patients, our prognostic model results showed that high-risk patients with no more than one adverse factor presented a 5-year survival rate of 85.9\%, but patients with three adverse factors had a 5-year survival rate of $43.0 \%$. Harrell's concordance index (C-index) of the risk group score was 0.768 . Therefore, the new prognostic model could be used to develop risk-adapted treatment approaches for adult sporadic BL.

Keywords: Sporadic Burkitt lymphoma, Adult, Pretreatment inflammatory biomarkers, Prognostic index, Real-word multicenter study

*Correspondence: huanghe@sysucc.org.cn; linty@sysucc.org.cn ${ }^{\dagger}$ Mei-ting Chen, Fei Pan, Yung-chang Chen, Wei Zhang and Hui-juan Lv contributed equally to this work.

${ }^{1}$ Department of Medical Oncology, Sun Yat-sen University Cancer Center, State Key Laboratory of Oncology in South China, Collaborative Innovation Center for Cancer Medicine, No. 651, Dongfeng East Road, Yuexiu District, Guangzhou 510060, China

Full list of author information is available at the end of the article

\section{Introduction}

Burkitt lymphoma (BL) is a rare but highly aggressive subtype of non-Hodgkin lymphoma (NHL) with the genetic hallmark of MYC gene translocation, including three different variants, namely, endemic, sporadic and immunodeficiency associated [1-3]. Endemic BL is 
associated with malaria and Epstein-Barr virus (EBV) [4]. The immunodeficiency-related variant has close relationship with human immunodeficiency virus (HIV) [5]. Sporadic $\mathrm{BL}$ is typically seen in young patients, but accounts for approximately $1 \%$ of adult NHLs [6].

With intense chemotherapy treatment, disease prognosis is excellent in children but poor in adults [7]. Acute treatment-related toxicity, such as severe myelosuppression, is an important factor affecting treatment outcomes in adults [8]. Mark Roschewski et al. demonstrated that dose-adjusted etoposide, doxorubicin, cyclophosphamide, vincristine, prednisone, and rituximab (DAEPOCH-R) could avoid the need for high-dose intensive chemotherapy in adults with BL [9]. The International Prognostic Index (IPI) is frequently used for prognostication in many aggressive lymphomas, but is not commonly used in BL [10]. According to the Murphy staging system, childhood BL can be staged and classified as low or high risk based on the number of involved sites, presence of bulky disease, and lactate dehydrogenase (LDH) [11]. However, there is no standardized prognostic model for adult BL. Recently, in a multicenter real-world study in Western countries presented that ages $\geq 40$, Eastern Cooperative Oncology Group (ECOG) performance status (PS) $\geq 2$, LDH 3 times more than the upper normal limit and central nervous system (CNS) involvement predicted inferior survival and constructed a BL-IPI in adult BL $[12,13]$. But the prognostic model for adult patients with sporadic BL in Asian patient is still under establishment.

There is evidence that the inflammatory response plays a key role in different stages of tumor development, including initiation, promotion, invasion and metastasis [14]. However, few studies have focused on inflammatory biomarkers in BL. Wang et al. showed that low lymphocyte to monocyte ratio (LMR) was an independently adverse prognostic factor in 62 adult patients with sporadic BL [15]. Thus, it is important to identify inflammatory biomarkers to estimate prognosis more precisely.

In the current study, we performed a multicenter retrospective analysis to identify inflammatory biomarkers to predict survival in 336 adult patients with sporadic BL. A novel prognostic model was constructed to provide additional information for physicians making decisions regarding treatment options.

\section{Materials and methods}

\section{Eligibility criteria and study population}

We retrospectively surveyed 229 adult patients with newly diagnosed sporadic BL who were treated from August 2008 to September 2019 at Sun Yat-sen University Cancer Center, Sichuan Cancer Hospital, West China Hospital and Sichuan Provincial People's Hospital in the training cohort. Eligible patients had histologically confirmed sporadic BL according to the 2008 World Health Organization (WHO) criteria, and complete clinical, laboratory, and follow-up data. All patients were confirmed diagnosed of BL by experienced pathologists. In order to avoid inclusion of high levels of B-cell lymphoma, all cases had been detected by fluorescence in situ hybridization for c-Myc, Bcl-2, and Bcl-6. Evaluation included standard laboratory tests, 18F-labeled fluorodeoxyglucose positron emission tomography (18F-FDG PET)computed tomography (CT) scans of the whole body, and bone marrow aspiration and biopsy. Their medical records were analyzed, a prognostic model for sporadic BL was constructed, and we validated the results in a validation cohort. The validation cohort was retrospectively recruited from Tianjin Medical University Cancer Institute, Affiliated Hospital of Qingdao University, Henan Cancer Hospital and Hunan Cancer Hospital in China. The study protocol was approved by the ethical committee of Sun Yat-sen University Cancer Center (approval number SZR2019-016). Informed consent was not required because this study was a retrospective report of cases, which is a retrospective analysis of clinical data with no relevant to human biological ethic problems. The need of informed consent was waived by the ethical committee of the Sun yat-sen University Cancer Center. The study protocol was approved by the Institutional Review Board of the Sun yat-sen University Cancer Center and the study was performed in accordance with the principles of the Declaration of Helsinki. All methods were performed in accordance with the relevant guidelines and regulations.

\section{Data collection}

The following baseline clinical information was extracted from electronic medical records (EMRs). Patient characteristics included age, sex, ECOG PS, Ann Arbor stage, B symptoms, pathological diagnosis, risk stratification, EBV-encoded DNAs, bone marrow status, and CT or magnetic resonance (MR) images of the neck, nasopharynx, abdomen, chest and pelvis or PET/CT of the entire body. Patients were assigned risk group according to the definition by Mead et al. [16]. Patients with low-risk had all of the following features: (1) normal LDH level, (2) Ann Arbor stage of I and completely resected abdominal lesion or single extra-abdominal mass $<10 \mathrm{~cm}$; all other patients were considered high-risk.

The platelet, lymphocyte, monocyte, and neutrophil counts, LDH, C-reactive protein (CRP) and albumin (ALB) were collected from the last blood test before treatment. We calculated the neutrophil-to-lymphocyte ratio (NLR), derived neutrophil-to-lymphocyte ratio (d-NLR) and LMR. OS rates were selected as primary 
endpoints. OS was defined as the time between the date of diagnosis and the date of death or last follow-up.

\section{Statistical analysis}

Categorical characteristics were compared using a chisquare test. PLT, LMR, NLR, d-NLR and LDH were calculated with receiver operating curves (ROC) to determine the optimal cutoff values and then dichotomized into 2 categories: less than, and greater than or equal to the cutoff values. The normal upper and lower limit for PLT was 100 to $350 \times 10^{9} / \mathrm{L}$ and the normal lower and upper limit for LDH was 120 to $250 \mathrm{U} / \mathrm{L}$. The optimal cut-off value of CRP and ALB are their clinical standard values. We applied the Kaplan-Meier method to perform survival analysis. Univariate Cox regression analyses and multivariate proportional hazards regression models were carried out to identify independent prognostic factors. All reported $P$-values were two-sided, and $P<0.05$ was considered to be statistically significant. Discrimination was measured by Harrell's concordance index (C-index), which quantifies the likelihood of two random patients. The patient who relapsed for the first time had a higher possibility of interest event. The $\mathrm{C}$-index was calculated by $R$ version 4.0.2 via the survival and design packages. All statistical analyses were carried out using SPSS version 25 and $R$ version 4.0.2.

\section{Results}

\section{Patient characteristics}

In the training cohort, 229 patients (153 male, 76 females; median age, 40 years [range 18-79]) met the inclusion criteria. Clinical features for all patients are summarized in Table 1. Fifty-seven patients (24.9\%) presented with B symptoms. Most of the patients (132 cases, $57.6 \%$ ) had advanced disease (Ann Arbor stages III-IV). A total of 107 patients had more than one extra-nodal involvement. According to the IPI score, a majority of the patients (137 cases, 59.8\%) were scored 2-5, and 92 patients (40.2\%) were scored $0-1$. One hundred fifty-three patients $(66.8 \%)$ were classified as high-risk group and 76 patients (33.2\%) was stratified as low-risk group. A total of 152 patients (66.4\%) received R-CODOX-M (rituximab, cyclophosphamide, vincristine, doxorubicin, highdose methotrexate) based first-line chemotherapy, and 25 patients $(10.9 \%)$ had received R-EPOCH (rituximab, etoposide, prednisolone, doxorubicin, cyclophosphamide and vincristine) chemotherapy. Twenty-eight patients (12.2\%) were treated with R-CHOP (rituximab, cyclophosphamide, vincristine, doxorubicin and prednisolone) and high dose methotrexate-based regimens, 17 patients (7.4\%) received R-Hyper CVAD (rituximab, cyclophosphamide, doxorubicin, vincristine and dexamethasone) as first line chemotherapy, and 7 patients (3.1\%) were treated with miscellaneous anthracycline-based regimens. Twenty-nine patients (12.7\%) and 9 patients (3.9\%) had received radiation therapy and autologous hematopoietic stem cell transplantation (ASCT), respectively. Details are shown in Table 1.

\section{Determination of cut-off values for serum markers}

The ROC was used to identify optimal cut-off values for indexes. The area under the curve is presented in Table 2. The AUC for LDH and PLT was 0.708 and 0.654, respectively. The optimal cut-off values of CRP and albumin levels were $10 \mathrm{mg} / \mathrm{L}$ and $40 \mathrm{mg} / \mathrm{L}$, respectively. By analyzing the specificity and sensitivity of each value, the optimal cutoff values of d-NLR, PLT and LDH were taken as 1.63, $254 \times 10^{9} / \mathrm{L}$ and $334 \mathrm{U} / \mathrm{L}$, respectively. Thus, patients were dichotomized into 2 categories: less than, and greater than or equal to the cut-off values.

\section{Univariate analysis and multivariate analysis}

Table 3 shows the results of univariate analysis of clinical variables considered predictors of OS. The following clinical factors significantly predicted poor survival in univariate analysis: $E C O G \geq 2$, bone marrow involvement, more than two extranodal involvement sites, advanced Ann Arbor stage (III/IV), IPI score $\geq 2$, high risk stratification, with $\mathrm{CRP} \geq 10 \mathrm{mg} / \mathrm{L}, \mathrm{ALB}<40 \mathrm{~g} / \mathrm{L}$, $\mathrm{LDH} \geq 334 \mathrm{U} / \mathrm{L}, \mathrm{PLT}<254 \times 10^{9} / \mathrm{L}$, and $\mathrm{d}-\mathrm{NLR}<1.6$. The univariate survival analysis for OS according to optimal cut-off values of ALB, LDH, PLT, d-NLR, CRP, IPI score, Ann Arbor stage, and risk stratification is shown in Fig. 1. Multivariate analysis was performed on clinical parameters related to shorter OS. We found that three variables maintained a negative prognostic influence on OS by using forward conditional Cox regression: $\mathrm{ALB}<40 \mathrm{~g} / \mathrm{L}(P=0.041$, hazard ratio (HR), $2.251 ; 95 \% \mathrm{CI}$, 1.034-4.902), LDH $\geq 334 \mathrm{U} / \mathrm{L}(P<0.001, \mathrm{HR}, 0.199 ; 95 \%$ CI, $0.081-0.488)$, and PLT $<254 \times 10^{9} / \mathrm{L}(P=0.038, \mathrm{HR}$, 2.261; 95\% CI, 1.047-4.886) (Table 3).

\section{Prognostic model construction and survival analysis}

Consequently, based on these 3 independent prediction factors $\left(\mathrm{ALB}<40 \mathrm{~g} / \mathrm{L}, \quad \mathrm{PLT}<254 \times 10^{9} / \mathrm{L}\right.$, and $\mathrm{LDH} \geq 334 \mathrm{U} / \mathrm{L}$ ) for $\mathrm{OS}$ in the multivariate analysis, a new prognostic model for all 229 patients was constructed by combining factors as follows: Group 1 (135 cases, 59\%), no more than one adverse factor; Group 2 (60 cases, 26.2\%) two adverse factors; and Group 3 (34 cases, $14.8 \%$ ), three adverse factors. (Fig. 2A). The new predictive model for BL effectively stratified patients by prognosis. The median OS of Group 1 and Group 2 was not reached, while the median OS in Group 3 was 17 months. The 5-year survival rates of Group 1, 2 and 3 were $88.1,72.4$, and $45 \%$, respectively $(P<0.0001)$. 
Table 1 Baseline characteristics of patients

\begin{tabular}{|c|c|c|}
\hline Characteristics & Training $(n=229)$ & Validation $(n=107)$ \\
\hline \multicolumn{3}{|l|}{ Age (years) } \\
\hline$<60$ & 192(83.8\%) & $85(79.4 \%)$ \\
\hline$\geq 60$ & $37(16.2 \%)$ & $22(20.6 \%)$ \\
\hline \multicolumn{3}{|l|}{ Gender } \\
\hline Male & $153(66.8 \%)$ & $72(67.3 \%)$ \\
\hline Female & $76(33.2 \%)$ & $35(32.7 \%)$ \\
\hline \multicolumn{3}{|l|}{ ECOG score } \\
\hline$<2$ & $168(73.4 \%)$ & $75(70.1 \%)$ \\
\hline$\geq 2$ & $61(26.6 \%)$ & $32(29.9 \%)$ \\
\hline \multicolumn{3}{|l|}{ Ann Arbor stages } \\
\hline$|-| \mid$ & $97(42.4 \%)$ & $50(46.7 \%)$ \\
\hline III-IV & $132(57.6 \%)$ & $57(53.3 \%)$ \\
\hline B symptoms & $57(24.9 \%)$ & $22(20.6 \%)$ \\
\hline \multicolumn{3}{|l|}{ Risk stratification } \\
\hline Low & $76(33.2 \%)$ & $41(38.3 \%)$ \\
\hline High & $153(66.8 \%)$ & $66(61.7 \%)$ \\
\hline Bone marrow involved & $36(15.7 \%)$ & $16(15 \%)$ \\
\hline Central nervous system (CNS) involved & $14(6.1 \%)$ & $5(4.7 \%)$ \\
\hline Lymph-node involvement & $111(48.5 \%)$ & $49(45.8 \%)$ \\
\hline \multicolumn{3}{|l|}{ Extranodal involvement sites } \\
\hline$<2$ & $122(53.3 \%)$ & $63(58.9 \%)$ \\
\hline$\geq 2$ & $107(46.7 \%)$ & $44(41.1 \%)$ \\
\hline EBV encoded small RNAs (EBERs)(+) & $48(21 \%)$ & $28(26.2 \%)$ \\
\hline ALB $<40 \mathrm{mg} / \mathrm{L}$ & $92(40.2 \%)$ & $58(54.2 \%)$ \\
\hline $\mathbf{C R P} \geq 10 \mathrm{mg} / \mathrm{L}$ & $101(43.7 \%)$ & $47(43.9 \%)$ \\
\hline $\mathrm{LDH} \geq 334 \mathrm{U} / \mathrm{L}$ & $87(38 \%)$ & $43(40.2 \%)$ \\
\hline $\mathbf{d}-\mathbf{N L R} \geq 1.6$ & $58(25.3 \%)$ & $34(31.8 \%)$ \\
\hline PLT $<254 \times 10^{9} / \mathrm{L}$ & $127(55.5 \%)$ & $54(50.5 \%)$ \\
\hline International Prognostic Indexs (IPI) score $\geq 2$ & $137(59.8 \%)$ & $61(57 \%)$ \\
\hline Radiation therapy & $29(12.7 \%)$ & $12(11.2 \%)$ \\
\hline ASCT & $9(3.9 \%)$ & $3(2.8 \%)$ \\
\hline CNS prophylaxis & 184(80.3\%) & $89(83.2 \%)$ \\
\hline Abdominal lesions & $137(59.8 \%)$ & $58(54.2 \%)$ \\
\hline \multicolumn{3}{|l|}{ Chemotherapy regimen } \\
\hline R-CODOX-M & $152(66.4 \%)$ & $69(64.5 \%)$ \\
\hline $\mathrm{R}-\mathrm{EPOCH}$ & $25(10.9 \%)$ & $8(7.5 \%)$ \\
\hline $\mathrm{R}-\mathrm{CHOP}$ & $28(12.2 \%)$ & $15(14 \%)$ \\
\hline R-Hyper-CVAD & $17(7.4 \%)$ & $12(11.2 \%)$ \\
\hline Others & $7(3.1 \%)$ & $3(2.8 \%)$ \\
\hline
\end{tabular}

Based on the risk stratification by Mead et al. [16], the subgroup analysis for high-risk and low-risk patients was shown in Fig. 2B and C. Our prognostic model results showed that the high-risk patients with no more than one adverse factor presented a 5-year survival rate of $85.9 \%$, but patients with three adverse factors in high-risk group revealed a 5-year survival rate of $43.0 \%$
$(P<0.0001)$. No significant difference was shown in low-risk patients (Fig. 2C). The C-index is 0.768 (95\%CI 0.705-0.830). According to the IPI score, low-and lowintermediate risk patients could not be distinguished $(P=0.8889)$ (Fig. 2D). Low-risk and intermediate-risk patients were also not distinguished based on BL-IPI score $(P=0.5045)$ (Fig. 2E). 
Table 2 The AUC values of the variable were calculated for OS. Optimal cutoff values for each inflammatory biomarker

\begin{tabular}{llll}
\hline Marker & AUC & $P$ & $\begin{array}{l}\text { Optimal } \\
\text { cutoff } \\
\text { value }\end{array}$ \\
\hline LDH & 0.708 & $<0.001$ & 334 \\
d-NLR & 0.622 & 0.013 & 1.6 \\
PLT & 0.654 & 0.002 & 254 \\
NLR & 0.59 & 0.066 & $/$ \\
LMR & 0.596 & 0.052 & \\
\hline
\end{tabular}

External validation and survival prediction

To validate our novel prognostic model, 107 patients from another four cancer centers were included. The characteristics of these patients are shown in Table 1. There was good consistency between the validation cohort and the training cohort. Three risk groups could also be predicted using the nomogram, and the 5-year survival rates of Groups 1, 2 and 3 were 90.5, 77.2, and $42.9 \%$, respectively $(P<0.0001)$ (Fig. $3 \mathrm{~A})$. In the subgroup analysis for high-risk patients (Fig. 3B), our prognostic model showed that the high-risk patients with no more than one adverse factor presented a 5-year survival rate of $87.1 \%$, but patients with three adverse factors in the

Table 3 Univariate and multivariate analyses of potential prognostic factors for OS

\begin{tabular}{|c|c|c|c|c|}
\hline \multirow[b]{2}{*}{ Characteristics } & \multicolumn{2}{|l|}{ Univariate analysis } & \multicolumn{2}{|c|}{ Multivariate analysis } \\
\hline & $\mathrm{HR}(95 \% \mathrm{Cl})$ & $\mathbf{P}$ & $\mathrm{HR}(95 \% \mathrm{Cl})$ & $\mathbf{P}$ \\
\hline \multicolumn{5}{|l|}{ Age } \\
\hline \multicolumn{5}{|l|}{$<60$} \\
\hline$\geq 60$ & 0.754(0.35-1.626) & 0.472 & $1.012(0.407-2.519)$ & 0.979 \\
\hline \multicolumn{5}{|l|}{ Gender } \\
\hline \multicolumn{5}{|l|}{ Male } \\
\hline Female & $0.502(0.24-1.046)$ & 0.066 & & \\
\hline \multicolumn{5}{|l|}{ ECOG score } \\
\hline \multicolumn{5}{|l|}{$<2$} \\
\hline$\geq 2$ & $4.457(1.973-10.069)$ & $<0.001$ & $0.323(0.101-1.031)$ & 0.056 \\
\hline \multicolumn{5}{|l|}{ Ann Arbor stages } \\
\hline \multicolumn{5}{|l|}{$|-| \mid$} \\
\hline III-IV & $2.744(1.316-5.723)$ & 0.007 & $1.287(0.362-4.572)$ & 0.696 \\
\hline B symptoms & $1.155(0.593-2.252)$ & 0.672 & $1.409(0.577-3.438)$ & 0.452 \\
\hline \multicolumn{5}{|l|}{ Risk stratification } \\
\hline \multicolumn{5}{|l|}{ Low } \\
\hline High & $5.214(1.863-14.594)$ & 0.002 & $0.113(0.024-0.519)$ & 0.005 \\
\hline Bone marrow involved & $2.256(1.158-4.395)$ & 0.017 & $2.136(0.799-5.71)$ & 0.13 \\
\hline CNS involved & $0.469(0.184-1.194)$ & 0.112 & & \\
\hline Lymph-node involvement & $1.081(0.594-1.966)$ & 0.799 & & \\
\hline \multicolumn{5}{|l|}{ Extranodal involvement sites } \\
\hline \multicolumn{5}{|l|}{$<2$} \\
\hline$\geq 2$ & $2.143(1.144-4.014)$ & 0.017 & $0.841(0.351-2.016)$ & 0.698 \\
\hline EBERs & $0.147(0.035-0.61)$ & 0.008 & $9.825(1.31-73.703)$ & 0.026 \\
\hline ALB $<40 \mathrm{mg} / \mathrm{L}$ & $0.387(0.208-0.718)$ & 0.003 & $2.251(1.034-4.902)$ & 0.041 \\
\hline $\mathrm{CRP} \geq 10 \mathrm{mg} / \mathrm{L}$ & $2.612(1.369-4.982)$ & 0.004 & $1.124(0.494-2.557)$ & 0.781 \\
\hline $\mathrm{LDH} \geq 334 \mathrm{U} / \mathrm{L}$ & $5.361(2.747-10.462)$ & $<0.001$ & $0.199(0.081-0.488)$ & $<0.001$ \\
\hline $\mathbf{d}-\mathbf{N L R} \geq 1.6$ & $2.71(1.487-4.939)$ & 0.001 & $0.501(0.246-1.019)$ & 0.056 \\
\hline $\mathbf{P L T}<254 \times 10^{9} / \mathrm{L}$ & $0.348(0.171-0.706)$ & 0.003 & $2.261(1.047-4.886)$ & 0.038 \\
\hline IPI score $\geq 2$ & $2.376(1.171-4.822)$ & 0.017 & 4.195(1.34-13.139) & 0.014 \\
\hline Radiation therapy & $0.928(0.391-2.203)$ & 0.866 & & \\
\hline ASCT & $0.393(0.054-2.857)$ & 0.356 & & \\
\hline CNS prophylaxis & $0.619(0.261-1.467)$ & 0.276 & & \\
\hline Abdominal lesions & $1.152(0.612-2.166)$ & 0.661 & & \\
\hline
\end{tabular}




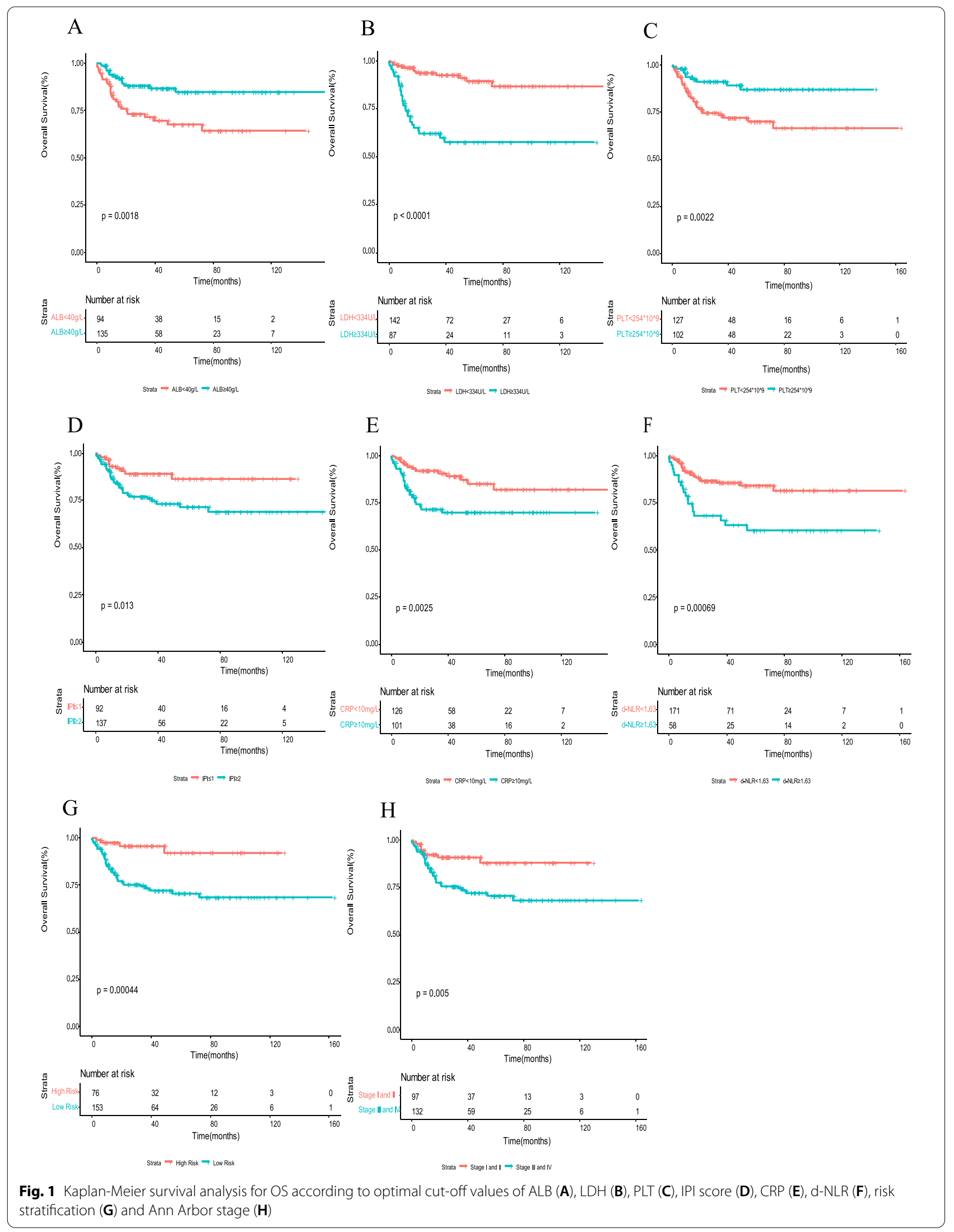




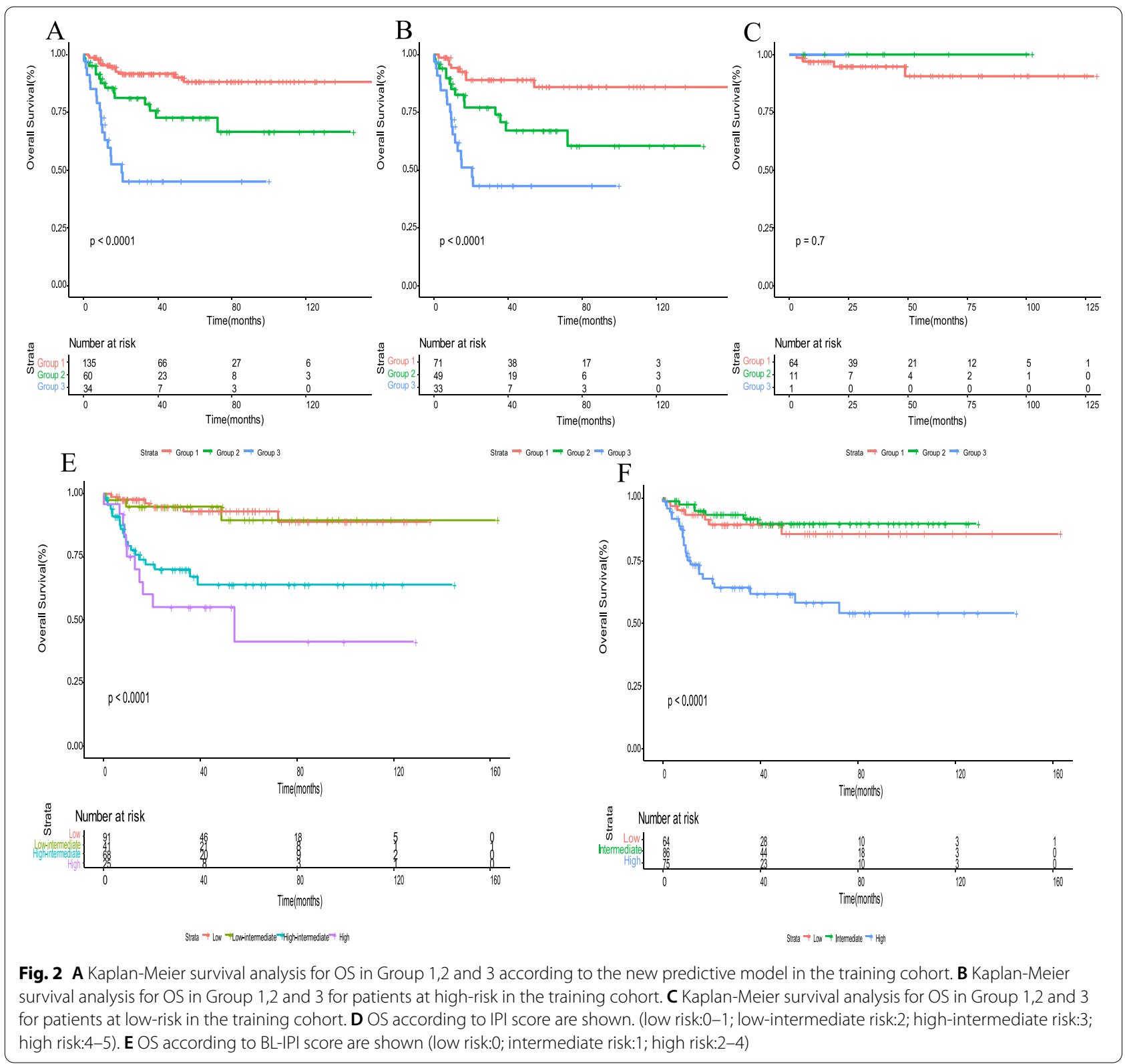

high-risk group revealed a 5-year survival rate of 38.9\% $(P=0.0013)$. The $\mathrm{C}$-index is 0.806 (95\%CI $0.727-0.887)$.

\section{Discussion}

In our study, we summarized the clinical characteristics and pretreatment inflammatory biomarkers in 336 adult patients with sporadic BL. It was demonstrated that platelets, albumin and LDH independently predicted survival in univariate and multivariate analyses. Based on the value of platelets, albumin and LDH, a novel prognosis model was constructed and demonstrated a strong ability to stratify risks. External validation was performed with this newly-developed prognostic model. To our knowledge, we are the first to report prognostic model of adult sporadic BL based on real-world multicenter study data in the Asian population.

When the immune system is activated, immune-related cells secrete proinflammatory cytokines, leading to systemic inflammation [17]. Systemic inflammation has been shown to be an etiologic factor of advanced cancer [18]. Recent studies have found that cancer-related inflammation plays a key role in the progression of different malignancies. Inflammatory biomarkers, such as white blood cell counts and acute phase proteins, have 
A
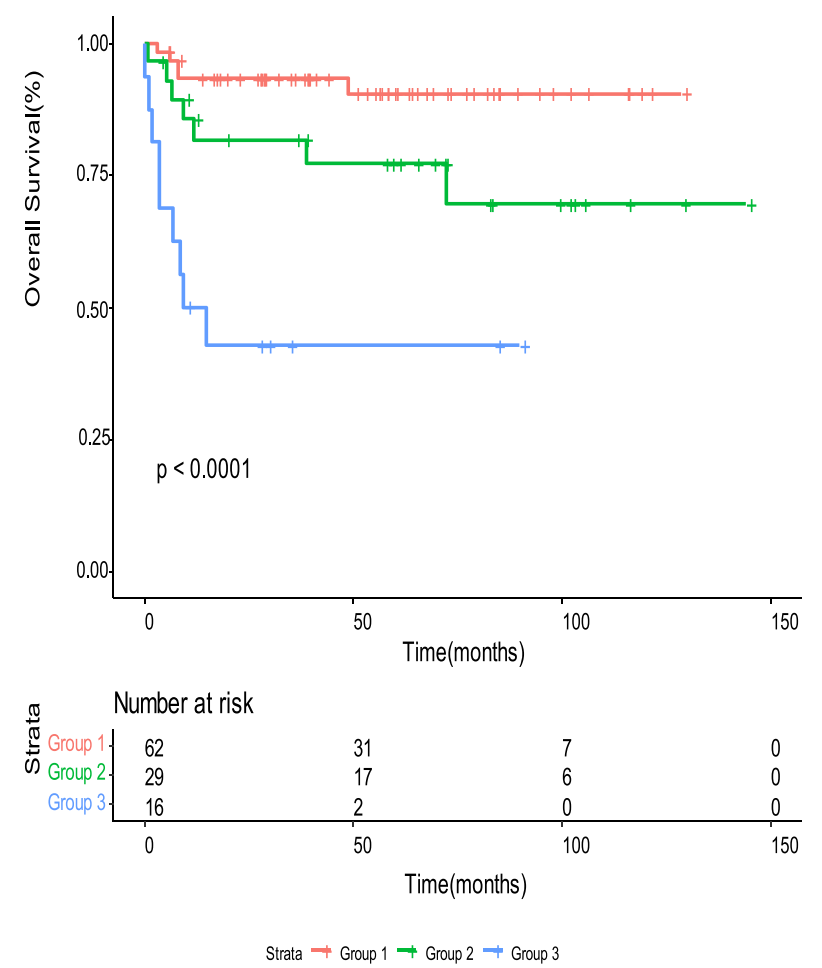

B

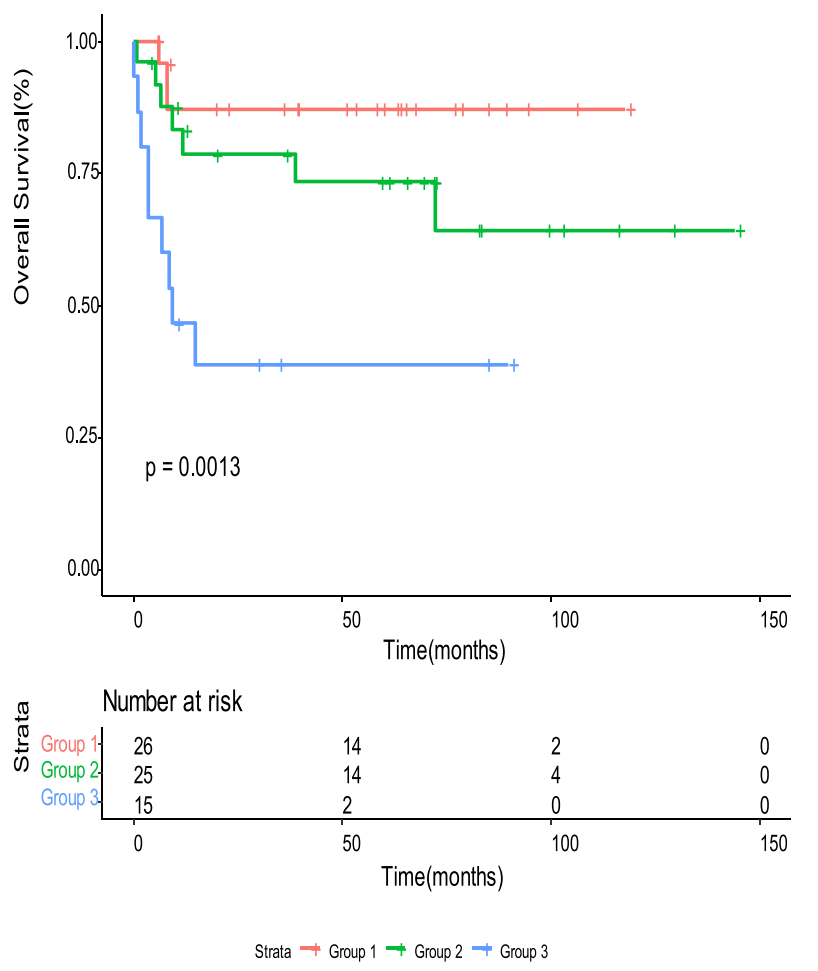

Fig. 3 A Kaplan-Meier survival analysis for OS in Group 1,2 and 3 according to the new predictive model in the validation cohort. B Kaplan-Meier survival analysis for OS in Group 1,2 and 3 for patients at high-risk in the validation cohort

repeatedly been shown to have prognostic value [19]. Prognostic markers of systemic inflammation, such as $\mathrm{d}-\mathrm{NLR}$, NLR and LMR, are associated with disease outcomes in a variety of tumors, and are readily available and inexpensive [20-27]. In our study, the variables of prognostic value were ALB, PLT and LDH, as independent indicators for sporadic BL. Our study revealed that in high-risk patients, the novel prognostic model could distinguish patients with significantly different survival rates. Patients at high-risk with no more than one adverse factor had a 5 -year survival rate of $85.9 \%$, which is similar to patients at low risk (5-year survival rate of 92.0\%). Thus, we suggest that patients with no more than one adverse factor might receive de-escalation chemotherapy to avoid severe side effects.

Several studies have found negative correlations between elevated LDH and survival in patients with BL $[28,29]$. The level of serum LDH in cancer patients has been reported to be an indicator of the cell proliferation rate and invasive potential [30]. In addition, LDH has been reported as a risk factor for tumor lysis syndrome (TLS) [31,32]. However, the proper cut-off value for LDH to predict outcome was not clear. In the risk stratification for BL [33], patients would be at high risk if LDH was above the upper normal limit. In Andrew M's study [34], LDH higher than 3 times the upper limit was associated with poor survival. Our study showed that $\mathrm{LDH} \geq 334 \mathrm{U} / \mathrm{L}$ predicted adverse outcomes.

Serum albumin levels, as an excellent indicator of malnutrition and cachexia, have been widely used in various advanced cancers [35]. Proinflammatory cytokines such as interleukin (IL)-6, IL-1, and tumor necrosis factor, which regulate the production of hepatocyte albumin, causing a lower serum albumin concentration [36, 37]. Platelets have the ability to cause cell adherence in infections, inflammation and many malignant tumors [38]. Furthermore, they also mediate tumor cell growth, proliferation, and angiogenesis. Activated platelets can interact with cancer cells through paracrine signaling or direct contact to promote tumor cell growth and survival $[39,40]$. In a recent study by Rachidi $\mathrm{S}$ et al., high platelet counts were associated with poor prognosis in patients with head and neck cancer, while patients with mid-normal platelet counts $\left(230-314 \times 10^{9} / \mathrm{L}\right)$ had a higher survival rate [41]. Suk-young Lee et al. analyzed 29 patients with intrahepatic cholangiocarcinoma who received 
gemcitabine plus cisplatin and found that platelet count $\leq 180 \times 10^{9} / \mathrm{L}$ was an independent prognostic factor for decreased OS [42]. In the current study, we found that platelet $<254 \times 10^{9} / \mathrm{L}$ predicted adverse outcomes. This suggests that lower platelet level might be a marker of poor prognosis associated with BL. Further research will focus on the relationship between platelet counts and different cancer survival rates.

Peripheral LMR predicts survival outcomes in some hematologic malignancies and solid tumors such as diffuse large B-cell lymphoma, bladder cancer, soft tissue sarcomas and gastric cancer [43-46]. Stotz and colleagues reported that a low LMR predicted poor clinical outcome in stage III colon cancer patients [47]. Porrata et al. found that patients with high peripheral blood LMR before and during treatment of classical Hodgkin's lymphoma had better OS [48]. Wang et al. showed that low LMR predicted poor outcome in 62 sporadic BL patients [15]. In our study, with a larger sample size, LMR was not associated with BL. Further study is need to confirm the underlying mechanism between LMR and BL. Proctor et al. reported that the d-NLR, which is based on leukocyte and neutrophil counts, had a prognostic value similar to that of the NLR. It could be recommended for risk stratification in chemotherapy patients [49]. The prognostic value of the d-NLR was further confirmed in subsequent studies in various types of cancers [50-52]. In our investigation, it was not associated with patient outcomes in multivariate analysis. We will expand the sample size to test our results in future studies.

In general, the IPI was shown to be a reliable prognostic indicator in aggressive lymphomas, taking into account the Ann Arbor stage, B symptoms, ECOG score, LDH and the extent of extranodal involvement, which were without significant prognostic value in our study. Similarly, the BL-IPI did not have a good risk stratification in our study. Because sporadic BL is considerably different from other aggressive lymphomas clinically and biologically, the IPI and BL-IPI might not be ideal for predicting OS in sporadic BL. Risk stratification has been widely used in BL patients, but the exploration of risk factors remains important. Mussolin et al. analyzed the long-distance polymerase chain reaction product in children with BL and developed a poor-prognosis subgroup among patients with high-risk BL [33]. In our study, we constructed a novel prognostic model for adult sporadic BL patients, including ALB, PLT and LDH, which was able to distinguish three groups with strikingly different survival rates.

Our study had some limitations of note. One limitation of this study lies in its retrospective nature and its heterogeneity in baseline risk and treatment factors, which may have led to potential bias. Furthermore, in the multivariate analysis, the prognostic values of tumor grade, CNS invasion and bone marrow invasion were not statistically significant, possibly due to the limited number of patients. Finally, these preliminary results needed to be further validated in larger prospective studies to clarify the mechanisms.

In summary, this study identifies pretreatment serum levels of ALB, PLT and LDH as clinically useful markers, which are inexpensive and readily available, to stratify patients into different risk groups that call for different treatments. Future studies involving larger patient groups with longer follow-up periods are needed to verify the results.

\section{Supplementary Information}

The online version contains supplementary material available at https://doi. org/10.1186/s12885-021-09144-1.

Additional file 1: Supplementary Table 1. The AUC values of the variable were calculated for PFS.

\section{Acknowledgments}

The authors thank all the patients, their families, and the institutions for supporting this study. They acknowledge all medical staff, staff nurses and research nurses, all of whom strongly contributed to the success of the study.

\section{Authors' contributions}

Tong-yu Lin and He Huang designed the study; Xiao-qian Li, Hua-wei Weng, Ze-geng Chen, Tao Pan, Ya-wen Wang, Hui-juan Lv, Yuan-yuan Shen, Li-min Chen, Yu-yi Yao and Xu-dong Li collected the data. Hui-lai Zhang, Zhao Wang, Yung-chang Chen, Wei Zhang, Xiao-jie Fang, Huang-ming Hong, Li-qun Zou, Hong-qiang Guo, Ke Xie, Hui Zhou, Hong-wei Xue assembled the data; Fei Pan and Mei-ting Chen analysed and interpreted the data; All authors wrote and gave final approval to the manuscript.

\section{Funding}

This work was supported by grants from the Natural Science Foundation of Guangdong Province (2019A1515010742).

Availability of data and materials

The datasets generated during the current study are available from the corresponding author on reasonable request.

\section{Declarations}

\section{Ethics approval and consent to participate}

The clinical data was acquired with the approval and permission of the Institutional Review Board of the Sun Yat-sen University Cancer Center. The study protocol was approved by the ethical committee of Sun Yat-sen University Cancer Center (approval number SZR2019-016) and the study was performed in accordance with the principles of the Declaration of Helsinki. Informed consent was not required because this study was a retrospective report of cases, which is a retrospective analysis of clinical data with no relevant to human biological ethic problems. The need of informed consent was waived by the ethical committee of the Sun yat-sen University Cancer Center.

All methods were performed in accordance with the relevant guidelines and regulations.

\section{Consent for publication}

Not applicable.

Competing interests

The authors declare that they have no competing interests. 


\section{Author details}

${ }^{1}$ Department of Medical Oncology, Sun Yat-sen University Cancer Center, State Key Laboratory of Oncology in South China, Collaborative Innovation Center for Cancer Medicine, No. 651, Dongfeng East Road, Yuexiu District, Guangzhou 510060, China. ${ }^{2}$ Sichuan Cancer Hospital \& Institue, Sichuan Cancer Center, School of Medicine, University of Electronic Science and Technology of China, Chengdu 610041, China. ${ }^{3}$ Departments of Lymphoma, Tianjin Medical University Cancer Institute and Hospital, National Clinical Research Center of Cancer, Key Laboratory of Cancer Prevention and Therapy, Tianjin's Clinical Research Center for Cancer, the Sino-US Center for Lymphoma and Leukemia Research, Tianjin, China. ${ }^{4}$ Department of Oncology, Affiliated Hospital of Qingdao University, Qingdao, Shandong 266003, P.R. China. ${ }^{5}$ Affiliated Cancer Hospital of Xiangya Medical School, Central South University / Hunan Cancer Hospital, Changsha 410013, China. ${ }^{6}$ Department of Medical Oncology, Cancer Center, West China Hospital of Sichuan University, Chengdu 610041, China. ${ }^{7}$ The Affiliated Cancer Hospital of Zhengzhou University, Henan Cancer Hospital, Zhengzhou, China. ${ }^{8}$ Department of Oncology, Sichuan Provincial People's Hospital, Chengdu, P.R. China.

Received: 13 May 2021 Accepted: 23 December 2021 Published online: 07 January 2022

\section{References}

1. Casulo C, Friedberg J. Treating Burkitt lymphoma in adults. Curr Hematol Malig Rep. 2015;10(3):266-71.

2. Mangani D, Roberti A, Rizzolio F, Giordano A. Emerging molecular networks in Burkitt's lymphoma. J Cell Biochem. 2013;114(1):35-8.

3. Castillo J, Nadeem O. Improving the accuracy in prognosis for Burkitt lymphoma patients. Expert Rev Anticancer Ther. 2014;14(2):125-7.

4. Orem J, Mbidde E, Lambert B, de Sanjose S, Weiderpass E. Burkitt's lymphoma in Africa, a review of the epidemiology and etiology. Afr Health Sci. 2007;7(3):166-75.

5. Atallah-Yunes S, Murphy D, Noy A. HIV-associated Burkitt lymphoma. Lancet Haematol. 2020;7(8):e594-600.

6. Mead G, Sydes M, Walewski J, Grigg A, Hatton C, Pescosta N, et al. An international evaluation of CODOX-M and CODOX-M alternating with IVAC in adult Burkitt's lymphoma: results of United Kingdom lymphoma group LY06 study. Ann Oncol. 2002;13(8):1264-74.

7. Costa L, Xavier A, Wahlquist A, Hill E. Trends in survival of patients with Burkitt lymphoma/leukemia in the USA: an analysis of 3691 cases. Blood. 2013;121(24):4861-6.

8. Hoelzer D, Walewski J, Döhner H, Viardot A, Hiddemann W, Spiekermann K, et al. Improved outcome of adult Burkitt lymphoma/leukemia with rituximab and chemotherapy: report of a large prospective multicenter trial. Blood. 2014;124(26):3870-9.

9. Roschewski M, Dunleavy K, Abramson J, Powell B, Link B, Patel P, et al. Multicenter study of risk-adapted therapy with dose-adjusted EPOCH-R in adults with untreated Burkitt lymphoma. J Clin Oncol. 2020;38(22):2519-29.

10. International Non-Hodgkin's Lymphoma Prognostic Factors Project. A predictive model for aggressive non-Hodgkin's lymphoma. N Engl J Med. 1993;329(14):987-94.

11. Murphy S. Classification, staging and end results of treatment of childhood non-Hodgkin's lymphomas: dissimilarities from lymphomas in adults. Semin Oncol. 1980;7(3):332-9.

12. Evens A, Danilov A, Jagadeesh D, Sperling A, Kim S, Vaca R, et al. Burkitt lymphoma in the modern era: real-world outcomes and prognostication across 30 US cancer centers. Blood. 2021;137(3):374-86.

13. Olszewski A, Jakobsen L, Collins G, Cwynarski K, Bachanova V, Blum K, et al. Burkitt lymphoma international prognostic index. J Clin Oncol. 2021;39(10):1129-38.

14. Grivennikov S, Greten F, Karin M. Immunity, inflammation, and cancer. Cell. 2010;140(6):883-99.

15. Wang $L$, Wang $H$, Xia ZJ, Huang HQ, Jiang WQ, Lin TY, et al. Peripheral blood lymphocyte to monocyte ratio identifies high-risk adult patients with sporadic Burkitt lymphoma. Ann Hematol. 2015;94(10):1645-54.

16. Mead G, Barrans S, Qian W, Walewski J, Radford J, Wolf M, et al. A prospective clinicopathologic study of dose-modified CODOX-M/IVAC in patients with sporadic Burkitt lymphoma defined using cytogenetic and immunophenotypic criteria (MRC/NCRI LY10 trial). Blood. 2008;112(6):2248-60

17. McMillan D. Cancer and systemic inflammation: stage the tumour and stage the host. Br J Cancer. 2013;109(3):529.

18. Martin H, Ohara K, Kiberu A, Van Hagen T, Davidson A, Khattak M. Prognostic value of systemic inflammation-based markers in advanced pancreatic cancer. Intern Med J. 2014;44(7):676-82.

19. Gabay C, Kushner I. Acute-phase proteins and other systemic responses to inflammation. N Engl J Med. 1999;340(6):448-54.

20. Li Y, Jiang W, Huang J, Xia Z, Huang H, Li Z. The Glasgow prognostic score (GPS) as a novel and significant predictor of extranodal natural killer/Tcell lymphoma, nasal type. Am J Hematol. 2013;88(5):394-9.

21. Li Z, Huang J, Xia Y, Sun J, Huang Y, Wang Y, et al. Blood lymphocyteto-monocyte ratio identifies high-risk patients in diffuse large B-cell lymphoma treated with R-CHOP. PLoS One. 2012;7(7):e41658.

22. Krishnamoorthy $R$, Reddy V. Hepatopancreatic amylase activity as a function of warm-adaptation in a fresh-water field crab. Experientia. 1968;24(10):1019-20.

23. Nishijima T, Muss H, Shachar S, Tamura K, Takamatsu Y. Prognostic value of lymphocyte-to-monocyte ratio in patients with solid tumors: a systematic review and meta-analysis. Cancer Treat Rev. 2015;41(10):971-8.

24. Takenaka Y, Oya R, Kitamiura T, Ashida N, Shimizu K, Takemura K, et al. Prognostic role of neutrophil-to-lymphocyte ratio in head and neck cancer: a meta-analysis. Head Neck. 2018;40(3):647-55.

25. Kano S, Homma A, Hatakeyama H, Mizumachi T, Sakashita T, Kakizaki T, et al. Pretreatment lymphocyte-to-monocyte ratio as an independent prognostic factor for head and neck cancer. Head Neck. 2017;39(2):247-53.

26. Piciucchi M, Stigliano S, Archibugi L, Zerboni G, Signoretti M, Barucca V, et al. The neutrophil/lymphocyte ratio at diagnosis is significantly associated with survival in metastatic pancreatic Cancer patients. Int J Mol Sci. 2017;18(4):730

27. Luo H, Ge H, Cui Y, Zhang J, Fan R, Zheng A, et al. Systemic inflammation biomarkers predict survival in patients of early stage non-small cell lung Cancer treated with stereotactic ablative radiotherapy - a single center experience. J Cancer. 2018;9(1):182-8.

28. Havelange V, Pepermans X, Ameye G, Théate I, Callet-Bauchu E, Barin C, et al. Genetic differences between paediatric and adult Burkitt lymphomas. Br J Haematol. 2016;173(1):137-44.

29. Pillon M, Mussolin L, Carraro E, Conter V, Aricò M, Vinti L, et al. Detection of prognostic factors in children and adolescents with Burkitt and diffuse large B-cell lymphoma treated with the AIEOP LNH-97 protocol. Br J Haematol. 2016;175(3):467-75.

30. López-Guillermo A, Montserrat E, Bosch F, Terol M, Campo E, Rozman C. Applicability of the international index for aggressive lymphomas to patients with low-grade lymphoma. J Clin Oncol. 1994;12(7):1343-8.

31. Wilson F, Berns J. Tumor lysis syndrome: new challenges and recent advances. Adv Chronic Kidney Dis. 2014;21(1):18-26.

32. McBride A, Westervelt P. Recognizing and managing the expanded risk of tumor lysis syndrome in hematologic and solid malignancies. J Hematol Oncol. 2012:5:75.

33. Mussolin L, Pillon M, d'Amore E, Conter V, Piglione M, Lo Nigro L, et al. Minimal disseminated disease in high-risk Burkitt's lymphoma identifies patients with different prognosis. J Clin Oncol. 2011;29(13):1779-84

34. Evens A, Danilov A, Jagadeesh D, Sperling A, Kim S, Vaca R, et al. Burkitt lymphoma in the modern era: real world outcomes and prognostication across 30 US Cancer centers. Blood. 2021;137(3):374-386

35. Ryan A, Power D, Daly L, Cushen S, Ní Bhuachalla Ë, Prado C. Cancer-associated malnutrition, cachexia and sarcopenia: the skeleton in the hospital closet 40 years later. Proc Nutr Soc. 2016;75(2):199-211.

36. McMillan D. Systemic inflammation, nutritional status and survival in patients with cancer. Curr Opin Clin Nutr Metab Care. 2009;12(3):223-6.

37. Gupta D, Lis C. Pretreatment serum albumin as a predictor of cancer survival: a systematic review of the epidemiological literature. Nutr J. 2010;9:69.

38. Huang Z, Liu W, Guo Q, Liu C. Platelet parameters and expression of platelet membrane glycoprotein in childhood acute lymphoblastic leukemia. Genet Mol Res. 2015;14(4):16074-89.

39. Monreal M, Fernandez-Llamazares J, Piñol M, Julian J, Broggi M, Escola $D$, et al. Platelet count and survival in patients with colorectal cancer--a preliminary study. Thromb Haemost. 1998;79(5):916-8. 
40. Sharma D, Brummel-Ziedins K, Bouchard B, Holmes C. Platelets in tumor progression: a host factor that offers multiple potential targets in the treatment of cancer. J Cell Physiol. 2014;229(8):1005-15.

41. Rachidi S, Wallace K, Day T, Alberg A, Li Z. Lower circulating platelet counts and antiplatelet therapy independently predict better outcomes in patients with head and neck squamous cell carcinoma. J Hematol Oncol. 2014;7:65.

42. Lee SY, Kim HS, Choi YJ, Park KH, Kim ST. A prognostic index to identify patients with intrahepatic Cholangiocarcinoma who could benefit from gemcitabine plus Cisplatin. Am J Ther. 2014;23(6).

43. LiY, Gu K, Pan Y, Jiao Y, Zhai Z. Peripheral blood lymphocyte/monocyte ratio at the time of first relapse predicts outcome for patients with relapsed or primary refractory diffuse large B-cell lymphoma. BMC Cancer. 2014;14:341.

44. Zhang G, Zhu Y, Luo L, Wan F, Zhu Y, Sun L, et al. Preoperative lymphocyte-monocyte and platelet-lymphocyte ratios as predictors of overall survival in patients with bladder cancer undergoing radical cystectomy. Tumour Biol. 2015;36(11):8537-43.

45. Szkandera J, Gerger A, Liegl-Atzwanger B, Absenger G, Stotz M, Friesenbichler J, et al. The lymphocyte/monocyte ratio predicts poor clinical outcome and improves the predictive accuracy in patients with soft tissue sarcomas. Int J Cancer. 2014;135(2):362-70.

46. Zhou X, Du Y, Xu J, Huang Z, Qiu T, Wang X, et al. The preoperative lymphocyte to monocyte ratio predicts clinical outcomes in patients with stage II/III gastric cancer. Tumour Biol. 2014;35(11):11659-66.

47. Stotz M, Pichler M, Absenger G, Szkandera J, Arminger F, SchaberlMoser $R$, et al. The preoperative lymphocyte to monocyte ratio predicts clinical outcome in patients with stage III colon cancer. Br J Cancer. 2014;110(2):435-40

48. Porrata L, Ristow K, Colgan J, Habermann T, Witzig T, Inwards D, et al. Peripheral blood lymphocyte/monocyte ratio at diagnosis and survival in classical Hodgkin's lymphoma. Haematologica. 2012;97(2):262-9.

49. Proctor M, McMillan D, Morrison D, Fletcher C, Horgan P, Clarke SJB. A derived neutrophil to lymphocyte ratio predicts survival in patients with cancer. Br J Cancer. 2012;107(4):695-9.

50. Absenger G, Szkandera J, Pichler M, Stotz M, Arminger F, Weissmueller M, et al. A derived neutrophil to lymphocyte ratio predicts clinical outcome in stage II and III colon cancer patients. 2013;109(2):395-400.

51. Colloca G, Venturino A, Guarneri DJCi, immunotherapy: Cll. Reduction of derived neutrophil-to-lymphocyte ratio after four weeks predicts the outcome of patients receiving second-line chemotherapy for metastatic colorectal cancer 2020.

52. Capone M, Giannarelli D, Mallardo D, Madonna G, Festino L, Grimaldi A, et al. Baseline neutrophil-to-lymphocyte ratio (NLR) and derived NLR could predict overall survival in patients with advanced melanoma treated with nivolumab. 2018;6(1):74.

\section{Publisher's Note}

Springer Nature remains neutral with regard to jurisdictional claims in published maps and institutional affiliations.

Ready to submit your research? Choose BMC and benefit from:

- fast, convenient online submission

- thorough peer review by experienced researchers in your field

- rapid publication on acceptance

- support for research data, including large and complex data types

- gold Open Access which fosters wider collaboration and increased citations

- maximum visibility for your research: over $100 \mathrm{M}$ website views per year

At BMC, research is always in progress.

Learn more biomedcentral.com/submissions 